\title{
Concurrent chemoradiation with carboplatin for locally advanced cervical cancer at high-risk for developing cisplatin-induced renal dysfunction
}

\author{
Lucely Cetina ${ }^{1}$, Alicia Garcia-Arias ${ }^{1}$, Maria de Jesus Uribe ${ }^{1}$, Alma Astorga ${ }^{1}$, \\ Myrna Candelaria ${ }^{1}$, Aida Mota ${ }^{2}$, Roque Guadarrama ${ }^{2}$, Lesbia Rivera ${ }^{2}$, \\ Jose Hinojosa $^{2}$ and Alfonso Dueñas-Gonzalez*3
}

\author{
Address: ${ }^{1}$ Division of Clinical Research, Instituto Nacional de Cancerología, México, ${ }^{2}$ Division of Radiotherapy, Instituto Nacional de \\ Cancerología, México and ${ }^{3}$ Unidad de Investigación Biomédica en Cáncer, IIB UNAM, INCAN México \\ Email: Alfonso Dueñas-Gonzalez* - alfonso_duenasg@yahoo.com \\ * Corresponding author
}

\author{
from 24th Annual Meeting of the National Cancer Institute of Mexico \\ Mexico City, Mexico. 14-17 February 2007 \\ Published: 5 February 2007 \\ BMC Cancer 2007, 7(SuppI I):AI7 doi:I0.II86/I47I-2407-7-SI-A I7 \\ This article is available from: http://www.biomedcentral.com//47I-2407/7/SI/AI7
}

(C) 2007 Cetina et al; licensee BioMed Central Ltd.

\section{Background}

Chemoradiation based on weekly cisplatin is the standard treatment for locally advanced cervical cancer; however, the nephrotoxic potential and the requirement for hydration of cisplatin somewhat restrains its use. We previously reported that the recommended dose of carboplatin to be used weekly with radiation was $133 \mathrm{mg} / \mathrm{m}^{2}$ (IJROBP 2003). Hence, we wanted to analyze our results of treatment with carboplatin chemoradiation which was adopted as routine treatment in our Institution for patients having high risk conditions to develop renal dysfunction by cisplatin.

\section{Materials and methods}

We reviewed the files of 85 patients with locally advanced cervical cancer who received radiation plus weekly carboplatin. The three main conditions that dictated the use of carboplatin instead of cisplatin in the patients analyzed were diabetes mellitus, high blood pressure and/or $>70$ years old. Treatment compliance, response rate and survival were analyzed.

\section{Results}

A total of 85 patients who received radiation and carboplatin were analyzed. Mean age was 58 years (range, 27-79 years). The majority of cases were squamous cell carcinoma (94\%), and distribution according to International
Federation of Gynecology and Obstetrics (FIGO) stage was as follows: IB2, 4.7\%, IIA, 8.2\%; IIB, 41.1\%, IIIA, $4.7 \%$ and IIIB, $38.8 \%$; there were only two IVA cases $(2.5 \%)$. Overall, $93 \%$ of patients completed external beam, and intracavitary low-dose rate brachytherapy. The majority of patients received five and six courses of weekly carboplatin, $40 \%$ and $38.8 \%$ respectively. Complete responses were achieved in $72(84.7 \%)$ patients, whereas 5 patients $(5.8 \%)$ had persistent and $8(9.4 \%)$ progressive disease. At median follow-up (20.8 months; range, 5-45 months) overall survival is $81.7 \%$.

\section{Conclusion}

Our results support the use of chemoradiation with six weekly applications of carboplatin at $133 \mathrm{mg} / \mathrm{m}^{2}$ during external radiation for routine management of locally advanced cervical cancer in patients who present high risk factors to develop renal dysfunction. 